\title{
A Prospective Cohort Study Comparing Medical Student Intubation on Cadaveric and Manikin Models Using the King Vision ${ }^{\mathrm{TM}}$ Videolaryngoscope and Direct Laryngoscopy
}

\author{
Thomas M. Triplett, Lynn P. Roppolo, Jeff Van Dermark, John Pease, Benjamin Cooper, Christopher Couch, \\ Scott Knepper, Brian Kendall and April R. Gorman
}

Emergency Medicine Department, University of Texas, USA

Submission: January 26, 2016; Published: May 3, 2016

*Corresponding author: Thomas M. Triplett, Emergency Medicine Department, UT Southwestern Medical Center, 5323 Harry Hines Blvd, Dallas, TX 75390-8579, USA, Email: thom.triplett@gmail.com

\begin{abstract}
Objective: Compare intubation by medical students with direct laryngoscopy (DL) versus King Vision ${ }^{T M}$ videolaryngoscope in training and cadaveric models.

Methods: 24 medical students with no experience intubating humans were randomized into two groups: DL first or King Vision ${ }^{\mathrm{TM}}$ videolaryngoscope first. Following a short training, techniques were practiced ona manikin model. Intubation success, ease of use, and one timed manikin intubation was recorded. Students crossed, repeated training and trial with other technique. Students then intubated 3 cadavers with their initial technique, crossed over to intubate these same cadavers using their second technique. Cormack-Lehane (C-L) view of the glottic opening, time to intubation, intubation success, ease of use, and preferred technique were recorded.

Results: In manikin intubation, success rate was not significantly different. Mean time to intubation using DL was 19.67 seconds, 11.03 seconds with King Vision ${ }^{\mathrm{TM}}$. The median "ease of use" for DL was 7; King Vision ${ }^{\mathrm{TM}}$ was 4 ( 0 easiest, 10 hardest). In cadaver model, the median C-L view was 2 for DL, 1 for King Vision ${ }^{T M}$. Success rates were $82 \%$ for DL and $93 \%$ for King Vision ${ }^{T M}$. The median "ease of use" for DL was 5 and King Vision ${ }^{\mathrm{TM}}$ was 4.

Conclusion: The King Vision ${ }^{\mathrm{TM}}$ videolaryngoscope had statistically significant improved visualization of the glottic opening, intubation success rate when compared to DL, and was rated easier to use. Given the portable nature, low cost, ease of use, and easy maintenance; the King Vision ${ }^{\mathrm{TM}}$ videolaryngoscope should be considered an excellent device for intubation training orany emergent setting where advanced airway management is required.
\end{abstract}

Keywords: Videolaryngoscope; Laryngoscopy; Airway management; Cadaver study; Manikin study; King Vision; King Vision; Endotracheal intubation; Medical student

\section{Introduction}

Orotracheal intubation is a critical skill required to manage patients with airway compromise or respiratory failure. Traditionally, direct laryngoscopy (DL) has been the standard method used to intubate patients. DL can be complicated by many factors that obstruct direct visualization such as pathology to the oropharynx (e.g. tumor) and limited mobility of the cervical spine [1-5]. Videolaryngoscopes (VL), which provide an indirect view of the glottis and vocal cords, have been in use as an adjunct to DL for over a decade $[2,6,7]$. Due to its ability to see "around the corner" via indirect visualization of the glottic opening, VL has been recommended for intubation in patients with limited neck mobility, airway anatomy that is difficult to visualize, and for training novice intubators in laryngoscopy [1,2,4-6,8-11]. The King Vision ${ }^{\text {TM }}$ VL is a relatively new, single unit, indirect videolaryngoscope that is lightweight, has a self contained highresolution camera, is battery operated, and has a curved blade with the option of a tube-guiding channel for easier intubation. Unique to the King Vision ${ }^{\mathrm{TM}}$ setting it apart from other VL is its low cost and portability relative to all other VL.

\section{Objective}

The purpose of this investigation was to compare timing and intubation success of the King Vision ${ }^{\mathrm{TM}}$ VL to DL in a manikin and cadaveric model by medical students with no prior intubation experience on human beings. Secondary outcomes were ease 
of use of DL and the King Vision ${ }^{\mathrm{TM}}$ VL in both the manikin and cadaver model as well as the Cormack-Lehane (C-L) view in cadaver model only as this is the model that best simulates real life intubating conditions.

\section{Material and Methods}

This study was approved by the Institutional Review Board at the University of Texas Southwestern. This was a randomized cross-over simulation study design in which 24 first year medical students with fewer than 5 manikin intubations, not previously employed in healthcare, and no prior intubation experience on human beings were randomized into two groups. Group 1 was initially trained in the use of DL followed by testing their ability to intubate with DL on a manikin model. Group 2 was initially trained in the use of the King Vision ${ }^{\mathrm{TM}}$ VL followed by testing their ability to intubate with this device on a manikin model. Then, each group crossed over to intubate with the other technique using the same training and testing as detailed below. All training was performed using an adult Laerdal ${ }^{\circledR}$ airway management trainer (www.laerdal.com).

All participants were recruited by campus wide email and received no compensation for participation other than intubation skills training. Exclusion criteria were extensive intubation experience, defined as 5 or more previous lifetime attempts, or any prior human intubation experience. After written informed consent was obtained, each participating student was required to review an 11-minute DL intubation video, read a 4-page tutorial on basic endotracheal intubation, and attend a 10-minute orientation on the King Vision ${ }^{\text {TM }}$ VL. Group 1practiced DL intubation using a Macintosh 4 laryngoscope on a manikin model and Group 2 practiced VL intubation using the King Vision ${ }^{\mathrm{TM}}$ VL with a channeled blade until each student demonstrated 3 successful intubations and felt comfortable with their assigned technique. Students then had a timed intubation on the same manikin that they practiced their assigned technique. The time to intubation and success rate was recorded. The time period started once the tip of the laryngoscope passed the lip of the manikin and ended once the student verbalized that the intubation was complete. A research assistant confirmed endotracheal tube placement in the manikin via direct visualization. After completing a timed intubation on their assigned technique, each student completed a written survey to assess their perceived ease of use for that intubation technique using a 0-10 scale with 10 being "very difficult" and 0 being "easy."The students then crossed over techniques and repeated the intubation training, testing and survey.

Students were then assigned to 3 of 6 cadavers, each intubating all 3 assigned cadavers with the first technique, then crossing over to intubate these same 3 cadavers using the other technique. Students were asked to assess the C-L view of the cadaveric glottic opening for each timed intubation with each technique. As described in 1984 by Drs. Cormack and
Lehane, the C-L view was graded as follows: 1) Grade 1, most of the glottis is visible, 2) Grade 2, only the posterior extremity of the glottis is visible, 3) Grade 3, no part of the glottis (only the epiglottisis visible), and 4) Grade 4, not even the epiglottis can be exposed [12]. The following information was recorded for the cadaver intubations: time to intubation as described above with the manikins, C-L view of the glottic opening, and proper endotracheal tube (ETT) placement. ETT placement was confirmed by direct observation of the ETT in the trachea through a window created in the cricothyroid membrane by investigators, who were blinded to the study group of the participant during the intubation procedure. The $\mathrm{C}-\mathrm{L}$ view was verbalized to the research assistant present at the time of each intubation. After completing intubation on the assigned cadavers, each student completed a written survey to assess their perceived ease of use of each intubation techniqueas mentioned above with the manikins. Study was limited to 24 participants due to time, availability, and materials constraints.

\section{Results}

A total of 24 first-year medical students (10 (42\%) male, 14 (58\%) female) were consented to participate in the study. The King Vision ${ }^{\mathrm{TM}}$ VL is abbreviated as VL in the results section for the purpose of simplicity.

\section{Manikin data}

There were 48 measured intubations performed by the students on manikins, $24 \mathrm{DL}$ and $24 \mathrm{VL}$. The intubation success rate was $92 \%(n=22)$ for DL and $96 \%(n=23)$ for VL, p-value $=0.50$ with Fisher's exact test. The mean time to intubation was 19.67 seconds using the DL and 11.03 seconds using VL, p-value $=0.015$ with Student's t-test, Table 1. Utilizing Wilcoxon Rank Sum test, the median ranking for "ease of use" was 7 for DL and 4 for VL on a 0 to 10 scale with 0 being the easiest to use and 10 being the most difficult, $p$-value $=0.003$, indicating that students thought the VL was easier to use, Figure 1 which has transformed the 0 to 10 scale into a larger scale on the vertical axis to better demonstrate the distribution of the data.

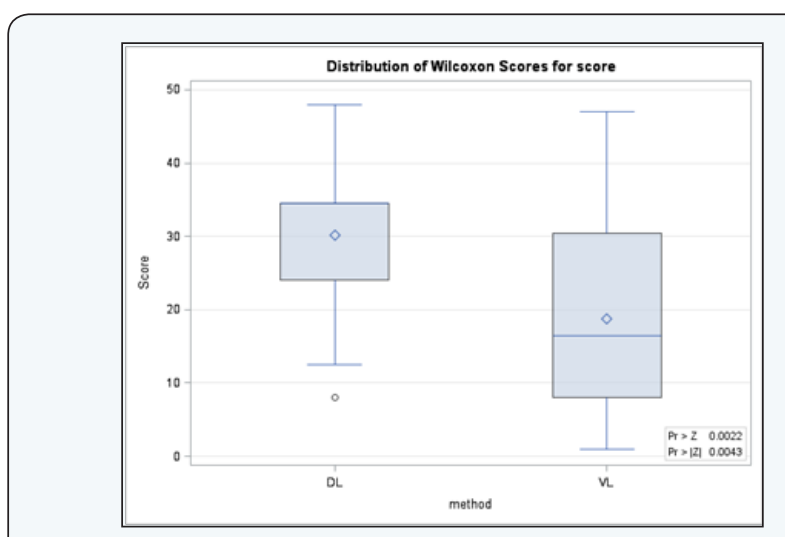

Figure 1: Non-parametric graph of transformed data demonstrating distribution of ease of use (lower score represents easier use). Manikin "Ease of Use". 
Table 1: Manikin and Cadaver Success rate, Time to intubation and Ease of use.

\begin{tabular}{|c|c|c|c|c|c|c|c|}
\hline & & Manikin & p-value & CI $95 \%$ & Cadaver & p-value & CI95\% \\
\hline \multirow{3}{*}{ Success Rate (\%) } & & & 0.50 & -0.18 to 0.09 & & 0.044 & -0.218 to -0.005 \\
\hline & DL & 92 & & & 82 & & \\
\hline & $\mathrm{VL}$ & 96 & & & 93 & & \\
\hline \multirow{3}{*}{ Mean Time (S) } & & & 0.015 & 1.74 to 14.9 & & 0.133 & -21.6 to 2.88 \\
\hline & DL & 19.67 & & & 27.11 & & \\
\hline & VL & 11.03 & & & 36.47 & & \\
\hline \multirow{3}{*}{$\begin{array}{c}\text { Ease of Use } \\
0 \text { being easiest }\end{array}$} & & & 0.003 & & & 0.048 & \\
\hline & DL & 7 & & & 5 & & \\
\hline & VL & 4 & & & 4 & & \\
\hline
\end{tabular}

DL: Direct Laryngoscopy

\section{VL: Video Laryngoscopy, King Vision ${ }^{\mathrm{TM}}$}

\section{Cadaver data}

There were 144 cadaveric intubation attempts (all 24 students intubated 3 different cadavers with each technique): 72 DL and 72 VL. The median C-L view grade was 2 for DL and 1 for VL, p-value < 0.001.Generalized Estimating Equations were utilized for our ordinal values of the C-L grade, Table 2. Eighteen of the intubation attempts were unsuccessful: $13 \mathrm{DL}$ and 5 VL. Success rates were $82 \%(59 / 72)$ for DL and $93 \%$ $(67 / 72)$ for VL, p-value $=0.044$, Table 1 . Generalized Estimating Equations were used as successful intubation was a binomial variable and confirmed that success rates were statistically higher with the VL method. No statistical difference was seen in the time to intubation for the method (DL vs. VL), Table 1. Repeated Measures Analysis of Variance was used to determine if the time to intubation varied between the DL and VL methods of intubation for all participants. The intubation technique that was used first by the student made no significant difference in time to intubation or success. "Ease of use" for the cadaver model utilizing the Wilcoxon Rank Sum test demonstrated that the VL was also easier to use in the cadaver, $\mathrm{p}=0.048$ Table 2 \& Figure 2. There was a positive trend in mean time from the first to third intubation with VL from 51.0s to 25.9 s. DL had no improvement with repeat intubation Table 3 \& Figure 3.

Table 2: Cormack-Lehane Scores on cadaver model.

\begin{tabular}{|c|c|c|}
\hline & \multicolumn{2}{|c|}{ Method } \\
\hline C-L Score & DL & VL \\
\hline 1 & 19 & 47 \\
\hline 2 & 31 & 18 \\
\hline 3 & 15 & 7 \\
\hline 4 & 4 & 0 \\
\hline
\end{tabular}

\section{DL: Direct Laryngoscopy}

VL: Video Laryngoscopy, King Vision ${ }^{\mathrm{TM}}$



Figure 2: Non-parametric graphs of transformed data demonstrating distribution of ease of use (lower score represents easier use). Cadaver "Ease of Use".

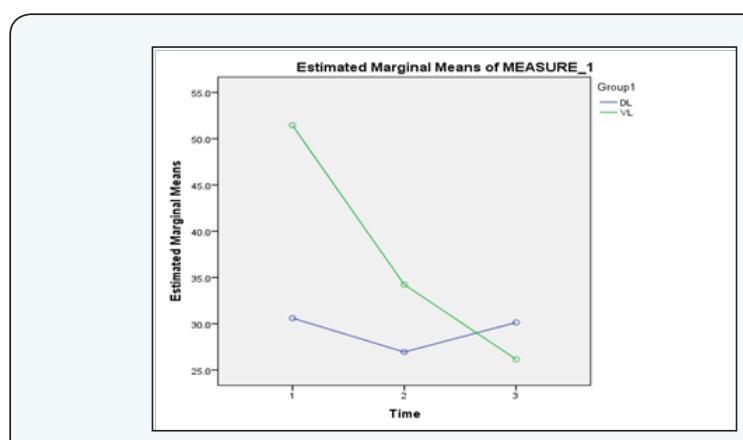

Figure 3: Progression of time to intubation with cadaver model.

Table 3: Progression of time to intubation with cadaver model.

\begin{tabular}{|c|c|c|}
\hline \multirow{2}{*}{ Attempt } & Mean Time (s) \\
\hline \multirow{3}{*}{ DL } & 1 & 27.5 \\
\cline { 2 - 3 } & 2 & 24.9 \\
\cline { 2 - 3 } & 3 & 29.4 \\
\hline \multirow{3}{*}{ VL VL } & 1 & 51 \\
\cline { 2 - 3 } & 2 & 32.1 \\
\cline { 2 - 3 } & 3 & 25.9 \\
\hline
\end{tabular}




\section{DL: Direct Laryngoscopy}

VL: Video Laryngoscopy, King Vision ${ }^{\mathrm{TM}}$

\section{Discussion}

The results of the current investigation clearly demonstrate that the King Vision ${ }^{\mathrm{TM}}$ VL has advantages compared to DL, consistent with other VL performance in the current literature. The King Vision ${ }^{\mathrm{TM}}$ was considered easier to use by participants, had a higher success rate and better C-L scores. However, when compared to other videolaryngoscopes, the King Vision ${ }^{\mathrm{TM}} \mathrm{VL}$ is less expensive, utilizes disposable blades, and is very portable.

The increased first pass success and C-L grade observed with King Vision ${ }^{\text {TM }}$ VL in this study are consistent with a 2014 study by Murphy et al. using novice paramedic students in a cadaver model. In this study, the King Vision ${ }^{\mathrm{TM}}$ VL had a higher success rate over DL, faster time to intubation, and better C-L grades [10]. Although our study showed insignificantly faster intubation times with DL in the cadaver model, the time to intubate decreased by almost 50 percent with successive attempts using the King Vision ${ }^{\mathrm{TM}} \mathrm{VL}$, indicating a rapid learning curve with the use of this device. In the current investigation, both glottic opening visualization and intubation success rates were significantly improved with the King Vision ${ }^{\mathrm{TM}}$ VL using the cadaveric model. Two meta-analyses of VL (GlideScope, X-Lite, Storz, McGrath, and Pentax-AWS) versus DL found equivocal results in success rate, but a significantly better view in difficult airways for VL. This is consistent with a study by Murphy comparing King Vision ${ }^{\text {TM }}$ to DL $[6,10,11]$. One frequent finding is that increased visualization of the glottic opening using VL may not proportionally translate to improved success or faster intubation times $[2,6,11,13,14]$. DL failures are typically due to inability to visualize glottic opening [14]. Most failures with VL are not from poor visualization but instead, are due to the inability to pass the ETT into the trachea [14-16]. This is often due to incongruence between the location of the glottic opening on VL and the trajectory of the ETT and is likely due to advancing the VL blade too distally, positioning of the rigid stylet used in the King Vision ${ }^{\mathrm{TM}}$ VL, or a very anteriorly placed glottis. Subtle maneuvers, equipment modifications and practice can reduce difficulties in passing the ETT. A Japanese meta-analysis showed significant improvement in success and time to success in VL (Airtraq and Pentax-AWS) with a channeled blade vs. DL [9], facilitating placement of the tip of the endotracheal tube much more anteriorly. The current investigation used a model of the King Vision ${ }^{\mathrm{TM}}$ VL with the channeled blade. Although we demonstrate a low failure rate consistent with most studies of King Vision VL, the most common reason observed for a King Vision $^{\mathrm{TM}}$ VL intubation failure is poor technique, which can be remedied using adequate training and briefly retraining if the device is rarely used [17].

The King Vision ${ }^{\mathrm{TM}}$ VL may used as a teaching tool for novice intubators similar to other $\mathrm{VL}$, and has advantages over them for training purposes due to its portable nature. Use of VL to train novice intubators has shown higher success rates using DL for endotracheal intubation in patients with normal airways as compared to training utilizing DL [5]. The shortcomings of DL for emergent intubators include significant training and practice to become competent with the direct laryngoscope and poor first-attempt tracheal intubation success rates [10]. The rapid progression in time to intubation with the King Vision ${ }^{\mathrm{TM}}$ VL seen in this data set is consistent with a study by Ayoub et al. which demonstrated a more rapid acclimatization to VL with a drastic decrease in time to intubation over 3 successive attempts when compared to DL on surgical patients undergoing anesthesia [5]. Several studies have demonstrated the ease of use of many videolaryngoscopes with a shorter learning curve as compared to DL $[5,13]$. In addition, any level user can easily gain skills in VL because glottic visualization is not diminished to the same degree, nor by the same factors that limit DL $[4,18]$. This is especially pronounced in the difficult airway setting with VL success of $76.4 \%$ compared to DL of $8.8 \%$ in one study [4]. In addition to steep learning curve and worse success with difficult airways, another pitfall of DL is the deterioration of skills if not frequently practiced. Murphy elaborates, 'even in larger institutions and systems, the opportunities to maintain intubation skill is limited, with a significant proportion of practitioners having one or no intubations per year' [10]. Having a device that is easy to use and portable makes continuing training easier to access and allows the retention of intubation skills even in a setting of infrequent intubation opportunities. Furthermore, it has been shown that training on manikins is an adequate strategy if events to train are rare or unpredictable [13]. Use of VL accelerates skill acquisition, can be used as the primary training technique, and can be used to retrain on manikins for infrequent practitioners with sufficient quality in training.

The King Vision ${ }^{\mathrm{TM}}$ VL has several clinical advantages over many other VL currently on the market in addition to ease of use and portability. Two case reports discuss the King Vision ${ }^{\mathrm{TM}}$ VL and its use clinically. One describes the success of King Vision ${ }^{\mathrm{TM}}$ VL for a difficult awake intubation of a patient with restricted mouth opening in a remote area with limited resources [19]. The King Vision ${ }^{\mathrm{TM}}$ VL was the only VL on the market that could have successfully intubated this person as no other VL has as narrow of a blade [19]. VL are also known for being easier to use than fiberoptic intubating scopes in most patients as the approach to intubating with VL is more similar to DL, the traditional method used for intubating. The second case series used the King Vision $^{\text {TM }}$ VL in awake intubations in patients with pathologic abnormalities in airway anatomy making normal visualization with standard DL impossible [17]. King Vision VL can be used in the pre hospital environment. Studies with paramedics in a simulated environment have shown King Vision ${ }^{\mathrm{TM}}$ VL is at least 
as good as and may be situationally more effective than DL under unusual circumstances such as, entrapped patients with both normal and difficult airways [10,20,21]. Other potential uses and future studies include unexpected in-hospital code situations on ward beds, international travel emergencies, public arenas, disaster scenarios, and pre-hospital intubation.

There were several limitations to the current investigation. The most obvious limitations were the small sample size and the simulated environment in which this study was conducted. In order to observe repeated measures data, ideally a repeat study will be performed with sample size of significant power to test the effects of different cadavers, the order in which the two methods were applied, and all interactions to better determine the factors that impact successful intubation. With the current preliminary study, we do not have the sample size needed to handle these more vigorous repeat measures analyses. We believe with a more concrete selection process, we will likely be able to confirm that, for most beginners, intubation using the King Vision ${ }^{\text {TM }}$ VL method will consistently demonstrate improved C-L views, success rates, and time to intubation. The King Vision ${ }^{\mathrm{TM}} \mathrm{VL}$ is a relatively new device and as of the writing of this article, a PubMed review did not identify any hospital based clinical trials completed, although a large Swiss multicenter trial protocol is written and currently ongoing with an objective of comparing 6 VL, 3 channeled blades (King Vision ${ }^{\mathrm{TM}}$, Airtraq $^{\mathrm{TM}}, \mathrm{A}$. P. Advance ${ }^{\mathrm{TM}}$ ) and 3 unchanneled blades (C-MAC ${ }^{\mathrm{TM}}$, GlideScope ${ }^{\mathrm{TM}}$, McGrath $^{\mathrm{TM}}$ ) in surgical intubation and an expected completion date of August 2015 [22].

\section{Conclusion}

The King Vision ${ }^{\mathrm{TM}}$ VL not only improves visualization of the glottic opening and intubation success rate, but it is easy to use. Although intubation times were insignificantly longer with the King Vision ${ }^{\mathrm{TM}}$ VL, cadavers were still successfully intubated rapidly and students demonstrated drastic improvement with successive intubations. Given the greater success rate of the King Vision $^{\mathrm{TM}} \mathrm{VL}$, the utility in intubating patients with a more difficult airway most likely parallels other videolaryngoscopes. It is a useful tool for training students and maintenance of intubating skills. Considering the portable nature, low cost, ease of use, and easy maintenance; the King Vision ${ }^{\mathrm{TM}}$ VL should be considered to be an excellent videolaryngoscopic device in any emergent setting where advanced airway management is required.

\section{Acknowledgements}

Ngosi, in memoriam.

\section{Support}

King Vision ${ }^{\mathrm{TM}}$ provided videolaryngoscopes and training technicians for this study. All materials returned promptly at end of study.

\section{References}

1. Kill C, Risse J, Wallot P, Seidl P, Steinfeldt T, et al. (2013) Videolaryngoscopy with glidescope reduces cervical spine movement in patients with unsecured cervical spine. J Emerg Med 44(4): 750-756.

2. Sakles JC, Mosier J, Chiu S, Cosentino M, Kalin L (2012) A comparison of the C-MAC video laryngoscope to the Macintosh direct laryngoscope for intubation in the emergency department. Ann Emerg Med 60(6): 739-748.

3. Kim HJ, Chung SP, Park IC, Cho J, Lee HS, et al. (2008) Comparison of the GlideScope video laryngoscope and Macintosh laryngoscope in simulated tracheal intubation scenarios. Emerg Med J 25(5): 279-282.

4. Phillips S, Celenza A (2011) Comparison of the Pentax AWS videolaryngoscope with the Macintosh laryngoscope in simulated difficult airway intubations by emergency physicians. Am J Emerg Med 29(8): 863-867.

5. Ayoub CM, Kanazi GE, Al Alami A, Rameh C, El-Khatib MF (2010) Tracheal intubation following training with the GlideScope compared to direct laryngoscopy. Anaesthesia 65(7): 674-678.

6. Su YC, Chen CC, Lee YK, Lee JY, Lin KJ (2011) Comparison of video laryngoscopes with direct laryngoscopy for tracheal intubation: a meta-analysis of randomised trials. Eur J Anaesthesiol 28(11): 788-795.

7. Doyle DJ (2009) A brief history of clinical airway management. Rev Mex Anestesiol 32(1): S164-S167.

8. Wang PK, Huang CC, Lee Y, Chen TY, Lai HY (2013) Comparison of 3 video laryngoscopes with the Macintosh in a manikin with easy and difficult simulated airways. Am J Emerg Med 31(2): 330-338.

9. Hirabayashi Y, Hoshijima H, Kuratani N (2013) Channelled videolaryngoscope: A meta-analysis of randomized controlled trials. Masui 62(7): 886-893.

10. Murphy LD, Kovacs GJ, Reardon PM, Law JA (2014) Comparison of the king vision video laryngoscope with the macintosh laryngoscope. J Emerg Med 47(2): 239-246.

11. Griesdale DE, Liu D, McKinney J, Choi PT (2012) Glidescope $\circledR^{\circledR}$ video-laryngoscopy versus direct laryngoscopy for endotracheal intubation: a systematic review and meta-analysis. Can J Anaesth 59(1): 41-52.

12. Cormack RS, Lehane J (1984) Difficult tracheal intubation in obstetrics. Anaesthesia 39(11): 1105-1111.

13. Rodriguez-Nunez A, Oulego-Erroz I, Perez-Gay L, Cortinas-Diaz J (2010) Comparison of the GlideScope Videolaryngoscope to the standard Macintosh for intubation by pediatric residents in simulated child airway scenarios. Pediatr Emerg Care 26(10): 726-729.

14. Sakles JC, Mosier JM, Chiu S, Keim SM (2012) Tracheal intubation in the emergency department: a comparison of GlideScope $®$ video laryngoscopy to direct laryngoscopy in 822 intubations. J Emerg Med 42(4): 400-405.

15. Carlson JN, Quintero J, Guyette FX, Callaway CW, Menegazzi JJ (2012) Variables Associated with Successful Intubation Attempts Using Video Laryngoscopy: A Preliminary Report in a Helicopter Emergency Medical Service. Prehosp Emerg Care 16(2): 293-298.

16. Mosier JM, Stolz U, Chiu S, Sakles JC (2012) Difficult airway management in the emergency department: GlideScope videolaryngoscopy compared to direct laryngoscopy. J Emerg Med 42(6): 629-634.

17. Gaszynska E, Gaszynski T (2014) The King Vision ${ }^{\mathrm{TM}}$ video laryngoscope for awake intubation: series of cases and literature review. Ther Clin Risk Manag 10: 475-478. 
18. Wetsch WA, Carlitscheck M, Spelten O, Teschendorf P, Hellmich M, et al. (2011) Success rates and endotracheal tube insertion times of experienced emergency physicians using five video laryngoscopes: a randomised trial in a simulated trapped car accident victim. Eur J Anaesthesiol 28(12): 849-858.

19. de Pinho MM, Bastos AM, Fontes CL, Pinto C, Adolfo BC (2014) Case report: tracheal intubation with King Vision in a patient with oral opening <1cm: 19AP1-7. Eur J Anaesthesiol 31(Supp 52): 270.

20. Gaszynska E, Gaszynski T (2014) Intubation by paramedics using the intubating laryngeal mask or AirTraq, King Vision and Macintosh laryngoscopes in vehicle-entrapped patients: a manikin study. Eur J Emerg Med 21(2): 152.
21. Burnett AM, Frascone RJ, Wewerka SS, Kealey SE, Evens ZN, et al. (2014) Comparison of success rates between two video laryngoscope systems used in a prehospital clinical trial. Prehosp Emerg Care 18(2): 231-238.

22. Theiler L, Hermann K, Schoettker P, Savoldelli G, Urwyler N, Kleine-Brueggeney M, et al. (2013) SWIVIT--Swiss video-intubation trial evaluating video-laryngoscopes in a simulated difficult airway scenario: study protocol for a multicenter prospective randomized controlled trial in Switzerland. Trials 14: 94. 